\title{
DEVELOPMENT OF OPTIMAL TOUR TIME MODEL FOR TOURIST DESTINATIONS IN A CITY: APPLICATION IN POKHARA CITY NETWORK
}

\author{
Purushottam Baral and Jagat Kumar Shrestha* \\ Civil Engineering Department, Pulchowk Campus, Institute of Engineering, Tribhuvan University \\ *E-mail:jagatshrestha@ioe.edu.np
}

\begin{abstract}
When a tourist visits a city, they tend to visit as many touristic places in the city as possible within their time limit. So, for optimization of their limited time budget and allowing them to spend their maximum time in the destination, this paper proposes a model to find an optimal route for the destination points a tourist is interested into. The computer model developed in this paper makes a sub-tour within the original network and search for an optimal tour route hence giving total travel time and total time required to visit desired destinations, and returning back to the place where you started from. The model enables fast decision support for tourists. The model developed is then applied in network of Pokhara and optimal route is found for a sample set of destinations.
\end{abstract}

KEYWORDS: Optimization, Point of Interest, Tourist destinations, Travelling Salesman Problem

\section{INTRODUCTION}

Nowadays, many tourists plan their vacation based on web sites, articles in magazines, or on guidebooks available in bookstores or libraries. These tourists visit a region or city during one or more days (P. Vansteenwegen and D. V. Oudheusden, 2007). So, they want to use their time in optimal way. During the pre-trip stage, tourists have to spend their time collecting information about the destination in order to start planning the stay. This is a difficult task because they have to choose the Point of Interests (POI) they are going to visit and estimate visits duration and traveling times (A. Garcia et al. 2010).

Tourist relies on travel agents, normal maps, and internet for planning their trips according to their POIs. For a city, tourist destination areas can be solved by Travelling Salesman Problem (TSP), where a salesman travels every house in a locality by travelling minimum distance and end at where it started. But, all tourists are not interested to visit all tourist destination areas within the city. So, the problem is not a TSP. The network containing POIs only becomes a sub-set for the original network of the city. For solving such Tourist Trip Design Problem, A mobile tourist guide (P. Vansteenwegen and D. V. Oudheusden, 2007) uses Orientation Problem (OP).

The OP is derived from an outdoor sport 
Orienteering. Orienteering is an outdoor sport usually played in a mountainous or heavily forested area. Armed with compass and map, a competitor starts at a specified control point, tries to visit as many other control points as possible within a prescribed time limit, and returns to a specified control point. Each control point has an associated score, so that the objective of orienteering is to maximize the total score [3]. In the OP case every location has a certain (attraction) value and the travel time between the points is known. However, not all the points can be visited since the total available time is limited. The OP is about determining a route, limited in time, between some of the points to maximize the total value (P. Vansteenwegen and D. V. Oudheusden, 2007).

The main difference between OP and TSP is that, in $\mathrm{OP}$ we have to maximize the total score collected, while in TSP we have to minimize the total distance to be travelled. In OP all vertices shouldn't be covered as in TSP. In OP, we have to find the shortest possible path between the selected vertices, but in TSP, we have to find shortest path for all vertices in the network.

Golden et al. (1987) prove that the OP is a Nondeterministic Polynomial-time Hard (NP-hard) problem. A problem is NP-hard if an algorithm for solving it can be translated into one for solving any NP-problem. So, for solving NPhard problem Heuristic approach should be applied. Heuristic, is any approach to problem solving, learning, or discovery that employs a practical method not guaranteed to be optimal or perfect, but sufficient for the immediate goals.

The Orienteering Problem (OP) can be modeled as a multi-level optimization problem. At the first level, we need to choose a subset of control points to visit. At the second level, we need to solve a Traveling Salesman Problem (TSP) or a shortest Hamiltonian path problem over the selected subset of control points I-M Chao et al. (1996). In fast and effective heuristic for the orienteering problem I-M Chao et al. (1996) used Greedy algorithm for solving OP. Keller (1989) treats the OP as a special case of the multi-objective vending problem in which the decision maker must trade off maximizing reward potential by visiting as many points as possible and minimizing travel cost by visiting as few points as possible.

\section{MODELLING THE PROBLEM}

A tourist TSP is a mathematical optimization problem that consists of a set of locations. The pair wise travel times between the locations are known. The goal is to find a tour that minimizes the total length during visiting the tourist destinations. The total tour (in route and time spent in POIs) cannot exceed the maximum amount of time the tourist has available.

Each tourist destination can be visited at most once. Hence, the problem can be a TSP consisting of the POIs for optimization of travel time in the network and time to be spent at POIs can be added to find the total tour time.

The problem has a network with a set of $\mathrm{N}$ vertices in a graph $\mathrm{G}=(\mathrm{V}, \mathrm{A})$ where $\mathrm{V}=\left\{\mathrm{v}_{1} \ldots\right.$ $\left.\mathrm{v}_{\mathrm{N}}\right\}$ is the vertex set $\mathrm{A}$ is the arc set. In this definition, the time to be spent $\mathrm{Tb}$ associated with each vertex $v_{1} \in V$ and the travel time is associated with each arc $\in$ A. In this problem 
v1 coincides with $\mathrm{v}_{\mathrm{N}}$. Using this notation introduced above, the problem is formulated as an integer problem. The following decision variables are used: $=1$ if a visit $i$ is following by a visit to vertex $\mathrm{j}, 0$ otherwise, defined only for $\mathrm{i}<\mathrm{j}$.

For a symmetric TSP $\left(t_{i j}=t_{j i}\right)$, the problem can be formulated as follows (Dantzig, George B. 1963):

Minimize:

$\mathrm{z}=\sum_{i}^{N} \sum_{j>i}^{N} t_{i j} x_{i j}$

Subject to:

$\sum_{j>i}^{N} x_{j i}+\sum_{j>i}^{N} t_{i j} x_{i j}=2 \quad \forall \mathrm{i}$

$\sum_{i, j \in \mathrm{S}} x_{i j} \leq|\mathrm{S}|-1 \quad \forall \mathrm{S} \subset \mathrm{N}$

$x_{i j} \in\{0,1\} \quad \forall \mathrm{i}, \mathrm{j}$

The objective function (1) is to minimize travelling time in the network. Constraints (2) ensure the connectivity of the path and guarantee that every vertex is visited at most once, Constraints (3) is necessary to prevent sub-tours. Constraint (4) shows the binary integrality. This formulation has a symmetric travel times between the vertices $\left(t_{i j}=t_{j i}\right)$. This corresponds to an undirected complete graph $\mathrm{G}$.

Finally, the following equation (5) gives the total tour time which is minimum travel time plus time to be spent in each node $i$. This time is to be compared with tie budget of the tourist $\left(\mathrm{T}<\mathrm{T}_{\max }\right.$, maximum time available for tourism. $\mathrm{T}=\mathrm{z}+\sum_{i}^{N} T_{i} \forall \mathrm{i}$

\section{SOLUTION APPROACH}

The Orienteering Problem (OP) can be modeled as a multi-level optimization problem. At the first level, we need to choose a subset of control points to visit. At the second level, we need to solve a Traveling Salesman Problem (TSP) or a shortest Hamiltonian path problem over the selected subset of control points (I-M. Chao et al. 1996). In fast and effective heuristic for the orienteering problem I-M. Chao et al.(1996) used Greedy algorithm for solving OP. Keller (1989) treats the OP as a special case of the multi-objective vending problem in which the decision maker must trade off maximizing reward potential by visiting as many points as possible and minimizing travel cost by visiting as few points as possible. The Orienteering Problem (OP) can be modeled as a multi-level optimization problem. At the first level, we need to choose a subset of control points to visit. At the second level, we need to solve a Traveling Salesman Problem (TSP) or a shortest Hamiltonian path problem over the selected subset of control points (I-M. Chao et al. 1996). In fast and effective heuristic for the orienteering problem I-M. Chao et al.(1996) used Greedy algorithm for solving OP. Keller (1989) treats the OP as a special case of the multi-objective vending problem in which the decision maker must trade off maximizing reward potential by visiting as many points as possible and minimizing travel cost by visiting as few points as possible. M. Mataija et al has solved TSP of 5 points using branch and bound method for delivering of packages at five randomly chosen addresses in the city. Laporte and Martello (1990) use a branch and bound method to solve small, randomly generated test problems that contain as many as 20 points. 
A mathematical model is developed for the Orienteering Problem (OP). Since, tourist are not interested in all the tourist destination, network of POIs for a tourist will be a subtour of original network. This model is then implemented to a sub-tour of the original road network. Here, the problem is reduced to a small TSP which is a sub-tour. This small network is taken by considering short distance between POIs. In this way, the tour utilize the intermediate nodes and edges of the network through which it would be shorter to reach to desired destination resulting to removal of some edge and nodes from original network.

Then a solution approach Branch and Bound is used to solve the problem. This will give a tour for the selected network with total time taken for the tour (travel time plus time spent in POIs). After that, we can compare this time to the time budget available to the tourist. If it is less than the time budget available for tourist, then the tour is ok. Otherwise we have to omit a node and try it again.

\section{APPLICATION OF THE MODEL IN POKHARA CITY NETWORK}

In this study for application of model, the network of Pokhara city is selected. Major POIs in Pokhara City are Fewa Lake, Mountain Museum, Regional Museum, Davis fall, Seti Gorge, Gupteshwor Cave, Bat Cave, Mahendra Cave, Bindabasini Temple, Tal Barahai Temple, Sarangkot and World Peace pagoda. All these tourist destination lies in the city as shown in figure 1. Each Destination is represented by a unique node number. This network shows 14 major tourist destinations in Pokhara.
The distance connecting the tourist destination is then collected. They act as weightage for each edge of the graph shown in figure 1. In this case, journey speed of $20 \mathrm{kmph}$ for plain city area and $10 \mathrm{kmph}$ for hilly area is used for estimating time required in minutes to cover distance. When two POIs are close by, time required to walk between these points is also considered. These distances are presented in Table 1 in the form of distance matrix.

The shortest path matrix (Table 2) shows only the least travelling time required in route after calculation using Floyd-Warshall algorithm. Time spent in each destination depends upon the interest of a tourist at that destination. For this test instance, time estimated in each tourist destination is shown in Table 3.

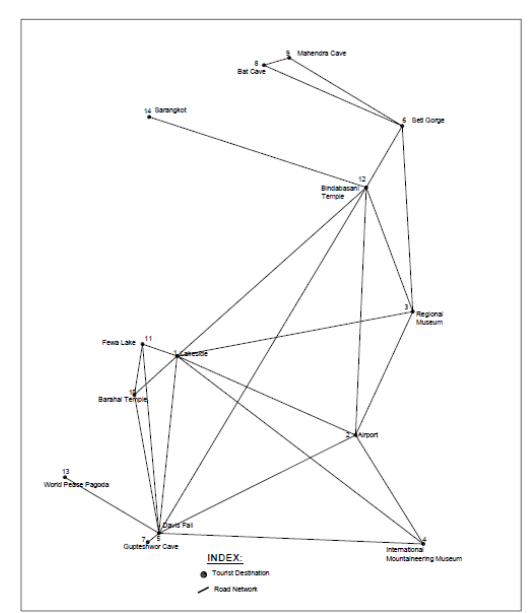

Figure 1: Major tourist Destination network in Pokhara city

The shortest path matrix (Table 2) shows only the least travelling time required in route after calculation using Floyd-Warshall algorithm. Time spent in each destination depends upon the interest of a tourist at that destination. For this test instance, time estimated in each tourist 
destination is shown in Table 3.

The shortest path matrix (Table 2) shows only the least travelling time required in route after calculation using Floyd-Warshall algorithm. Time spent in each destination depends upon the interest of a tourist at that destination. For this test instance, time estimated in each tourist destination is shown in Table 3.

Table 1: Distance Matrix (time in minutes)

\begin{tabular}{|l|c|c|c|c|c|c|c|c|c|c|c|c|c|c|c|}
\hline \multicolumn{1}{|c|}{ Destination } & & $\mathbf{1}$ & $\mathbf{2}$ & $\mathbf{3}$ & $\mathbf{4}$ & $\mathbf{5}$ & $\mathbf{6}$ & $\mathbf{7}$ & $\mathbf{8}$ & $\mathbf{9}$ & $\mathbf{1 0}$ & $\mathbf{1 1}$ & $\mathbf{1 2}$ & $\mathbf{1 3}$ & $\mathbf{1 4}$ \\
\hline Lakeside & $\mathbf{1}$ & 0 & 10 & 15 & 15 & 15 & 0 & 0 & 0 & 0 & 10 & 10 & 15 & 0 & 0 \\
\hline Airport & $\mathbf{2}$ & 10 & 0 & 10 & 10 & 10 & 0 & 0 & 0 & 0 & 0 & 0 & 15 & 0 & 0 \\
\hline Regional Museum & $\mathbf{3}$ & 15 & 10 & 0 & 0 & 0 & 10 & 0 & 0 & 0 & 0 & 0 & 10 & 0 & 0 \\
\hline Mountaineering Museum & $\mathbf{4}$ & 15 & 10 & 0 & 0 & 15 & 0 & 0 & 0 & 0 & 0 & 0 & 0 & 0 & 0 \\
\hline Davis Fall & $\mathbf{5}$ & 15 & 10 & 0 & 15 & 0 & 0 & 10 & 0 & 0 & 15 & 15 & 25 & 30 & 0 \\
\hline Seti Gorge & $\mathbf{6}$ & 0 & 0 & 10 & 0 & 0 & 0 & 0 & 10 & 10 & 0 & 0 & 5 & 0 & 0 \\
\hline Gupteshwor Cave & $\mathbf{7}$ & 0 & 0 & 0 & 0 & 10 & 0 & 0 & 0 & 0 & 0 & 0 & 0 & 0 & 0 \\
\hline Bat Cave & $\mathbf{8}$ & 0 & 0 & 0 & 0 & 0 & 10 & 0 & 0 & 10 & 0 & 0 & 0 & 0 & 0 \\
\hline Mahendra Cave & $\mathbf{9}$ & 0 & 0 & 0 & 0 & 0 & 10 & 0 & 10 & 0 & 0 & 0 & 0 & 0 & 0 \\
\hline Barahai Temple & $\mathbf{1 0}$ & 10 & 0 & 0 & 0 & 15 & 0 & 0 & 0 & 0 & 0 & 10 & 0 & 0 & 0 \\
\hline Fewa Lake & $\mathbf{1 1}$ & 10 & 0 & 0 & 0 & 15 & 0 & 0 & 0 & 0 & 10 & 0 & 0 & 0 & 0 \\
\hline Bindabasani Temple & $\mathbf{1 2}$ & 20 & 15 & 10 & 0 & 25 & 5 & 0 & 0 & 0 & 0 & 0 & 0 & 0 & 45 \\
\hline World Peace Pagoda & $\mathbf{1 3}$ & 0 & 0 & 0 & 0 & 30 & 0 & 0 & 0 & 0 & 0 & 0 & 0 & 0 & 0 \\
\hline Sarangkot & $\mathbf{1 4}$ & 0 & 0 & 0 & 0 & 0 & 0 & 0 & 0 & 0 & 0 & 0 & 45 & 0 & 0 \\
\hline
\end{tabular}

Table 2: Short Path Matrix (time in minutes)

\begin{tabular}{|l|c|c|c|c|c|c|c|c|c|c|c|c|c|c|c|}
\hline \multicolumn{1}{|c|}{ Destination } & & $\mathbf{1}$ & $\mathbf{2}$ & $\mathbf{3}$ & $\mathbf{4}$ & $\mathbf{5}$ & $\mathbf{6}$ & $\mathbf{7}$ & $\mathbf{8}$ & $\mathbf{9}$ & $\mathbf{1 0}$ & $\mathbf{1 1}$ & $\mathbf{1 2}$ & $\mathbf{1 3}$ & $\mathbf{1 4}$ \\
\hline Lakeside & $\mathbf{1}$ & 0 & 10 & 15 & 15 & 15 & 25 & 25 & 35 & 35 & 10 & 10 & 15 & 45 & 65 \\
\hline Airport & $\mathbf{2}$ & 10 & 0 & 10 & 10 & 10 & 20 & 20 & 30 & 30 & 20 & 20 & 15 & 40 & 60 \\
\hline Regional Museum & $\mathbf{3}$ & 15 & 10 & 0 & 20 & 20 & 10 & 30 & 20 & 20 & 25 & 25 & 10 & 50 & 55 \\
\hline Mountaineering Museum & $\mathbf{4}$ & 15 & 10 & 20 & 0 & 15 & 30 & 25 & 40 & 40 & 25 & 25 & 25 & 45 & 70 \\
\hline Davis Fall & $\mathbf{5}$ & 15 & 10 & 20 & 15 & 0 & 30 & 10 & 40 & 40 & 15 & 15 & 25 & 30 & 70 \\
\hline Seti Gorge & $\mathbf{6}$ & 25 & 20 & 10 & 30 & 30 & 0 & 40 & 10 & 10 & 35 & 35 & 5 & 60 & 50 \\
\hline Gupteshwor Cave & $\mathbf{7}$ & 25 & 20 & 30 & 25 & 10 & 40 & 0 & 50 & 50 & 25 & 25 & 35 & 40 & 80 \\
\hline Bat Cave & $\mathbf{8}$ & 35 & 30 & 20 & 40 & 40 & 10 & 50 & 0 & 10 & 45 & 45 & 15 & 70 & 60 \\
\hline Mahendra Cave & $\mathbf{9}$ & 35 & 30 & 20 & 40 & 40 & 10 & 50 & 10 & 0 & 45 & 45 & 15 & 70 & 60 \\
\hline Barahai Temple & $\mathbf{1 0}$ & 10 & 20 & 25 & 25 & 15 & 35 & 25 & 45 & 45 & 0 & 10 & 30 & 45 & 75 \\
\hline Fewa Lake & $\mathbf{1 1}$ & 10 & 20 & 25 & 25 & 15 & 35 & 25 & 45 & 45 & 10 & 0 & 30 & 45 & 75 \\
\hline Bindabasani Temple & $\mathbf{1 2}$ & 20 & 15 & 10 & 25 & 25 & 5 & 35 & 15 & 15 & 30 & 30 & 0 & 55 & 45 \\
\hline World Peace Pagoda & $\mathbf{1 3}$ & 45 & 40 & 50 & 45 & 30 & 60 & 40 & 70 & 70 & 45 & 45 & 55 & 0 & 100 \\
\hline Sarangkot & $\mathbf{1 4}$ & 65 & 60 & 55 & 70 & 70 & 50 & 80 & 60 & 60 & 75 & 75 & 45 & 100 & 0 \\
\hline
\end{tabular}

For testing the model developed, let us assume a tourist has only a day $\left(\mathrm{T}_{\max }=8\right.$ hours) for visiting in Pokhara. Depending upon his/her interest, s/he can design a trip based upon the information in figure 1, Table 1, Table 2 and Table 3. For example, the tourist stays at a Hotel in Lakeside and wants to visit Regional Museum, International Mountain Museum, Seti Gorge, Bindabasini Temple and Sarangkot, and return back to hotel. According to the Figure 1, the nodes to be covered are 1, 3, 4, 6, 12 and 14 as shown in Figure 2(a). It now becomes the reduced TSP.

Table 3: Spending Time at Tourist Destination

\begin{tabular}{|l|l|l|l|}
\hline Destination & Node & $\begin{array}{l}\text { M i i m u m } \\
\text { time Spent } \\
\text { (in min) }\end{array}$ & $\begin{array}{l}\text { Range of } \\
\text { Time Spent }\end{array}$ \\
\hline Lakeside & 1 & - & $2-8 \mathrm{hrs}$ \\
\hline Airport & 2 & - & - \\
\hline Regional Museum & 3 & 60 & $1-3 \mathrm{hrs}$ \\
\hline Mountaineering Museum & 4 & 120 & $2-6 \mathrm{hrs}$ \\
\hline Davis Fall & 5 & 60 & $1-2 \mathrm{hrs}$ \\
\hline Seti Gorge & 6 & 30 & $30 \mathrm{~min}-1 \mathrm{hrs}$ \\
\hline Gupteshwor Cave & 7 & 60 & $1-2 \mathrm{hrs}$ \\
\hline Bat Cave & 8 & 60 & $1-2 \mathrm{hrs}$ \\
\hline Mahendra Cave & 9 & 60 & $1-2 \mathrm{hrs}$ \\
\hline Barahai Temple & 10 & 60 & $1-3 \mathrm{hrs}$ \\
\hline Fewa Lake & 11 & 90 & $\begin{array}{l}1-8 \\
(1 \mathrm{day})\end{array}$ \\
\hline Bindabasani Temple & 12 & 60 & $1-4 \mathrm{hrs}$ \\
\hline World Peace Pagoda & 13 & 120 & $2-6 \mathrm{hrs}$ \\
\hline Sarangkot & 14 & 90 & $\begin{array}{l}1-8 \\
(1 \mathrm{day})\end{array}$ \\
\hline
\end{tabular}

The solution obtained by applying $B \& B$ algorithm is 1-12-14-6-3-4-1 (Figure 2(b)). Here, total time taken during travel is 155 minutes and total time taken for the whole tour is 515 minutes ( 8 hour 35 minutes) which exceeds $\mathrm{T}_{\text {max. }}$ Hence, it is not the feasible solution. So, $\mathrm{s} /$ he may drop one POIs (eg. Seti Gorge, node 6). Now, the network is further reduced to $1,3,4$, 12 and 14 (Figure 3(a)). Solving this network, we get the route 1-12-14-3-4-1 as shown in Figure 3(b). Here, total travel time is 140 minutes and total tour time is 480 minutes ( 8 hour 00 minutes) which is feasible solution in the time budget.

The network shown in Figure 1 is a reduced network of POIs from the original network. 
Although other node and edge are omitted from the network, it still has properties of the original network. Here the edge of the graph is the shortest distance between the POIs. For example, distance from 4 to 3 is 20 minutes, which is the sum of distance from 4-2 and 2-3 although node 2 is not seen in the reduced network.

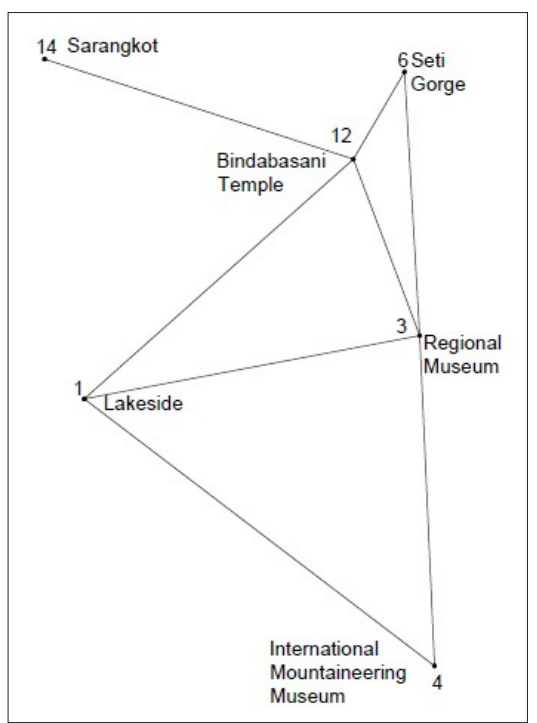

(a) Network with POIs 1, 3, 4, 6 12, 14

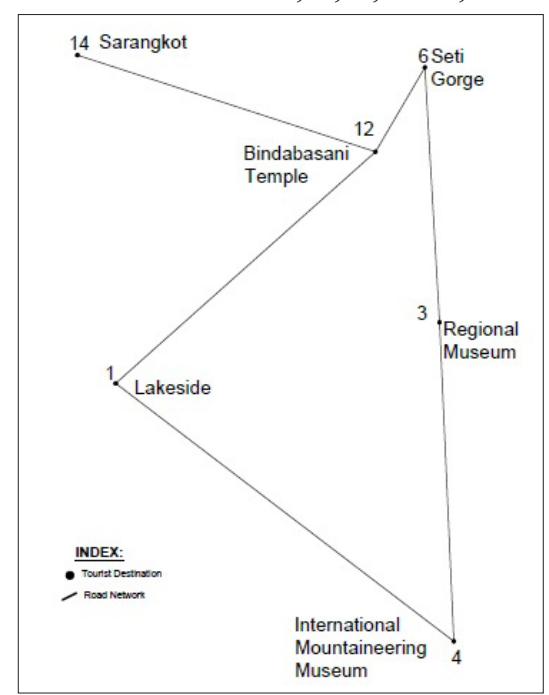

(b) Solution 1-12-14-6-3-4-1

Figure 2: Reduced Network with POIs 1, 3, 4,

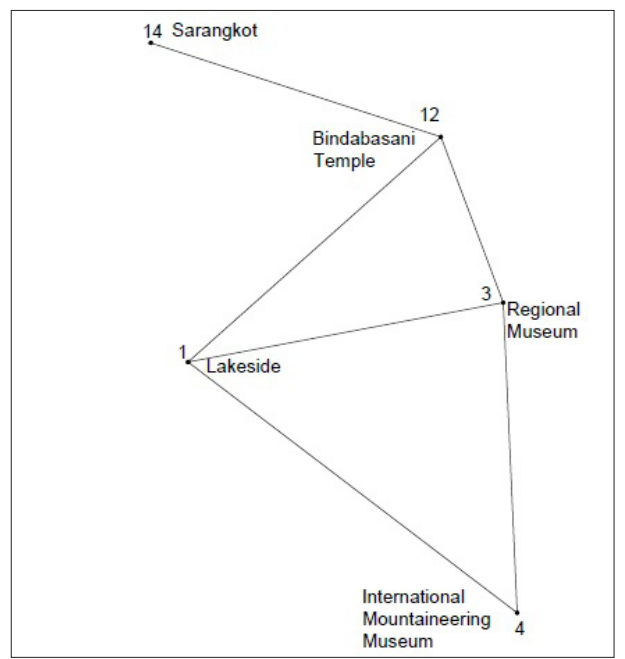

(a) Network with POIs 1, 3, 4, 12, 14

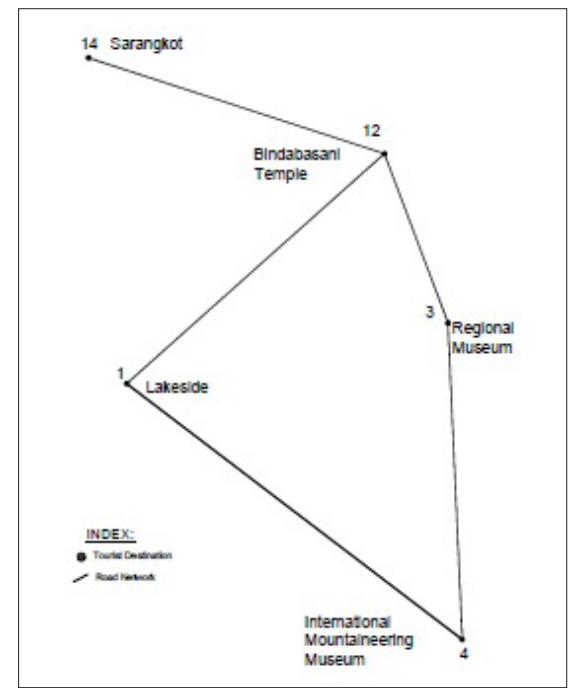

(b) Solution 1-12-14-3-4-1

Figure 3: Reduced network with POIs 1, 3, 4,

$$
12,14
$$

Apart from this example of just one day, if a tourist has 2 or more days, then they can plan trip with different set of POIs for different day. They can compare travel time for different set of POIs and find an optimal set of destination points for different day and spent most of the time in destination rather than spend time 
travelling between them.

In the example, node 1 (Lakeside) is taken as starting and ending point, but tourist also can plan their trip starting and ending from any other node convenient to them.

\section{CONCLUSIONS AND RECOMMENDATION}

A tourist new to city may not know the location of the POIs in the city, hence cannot plan the trip effectively. This paper has developed a model which helps to find an optimized route for visiting desired set of tourist destination in a city. This model takes a sub-tour of POIs and solves the network as a TSP and find an optimal route. Here a bigger network is reduced to smaller network by removing the nodes and edges, thus reducing the complicity of the problem. The reduced network still inherits the properties of original network and find shortest path from the whole network. A heuristic algorithm Branch and Bound is used for solving the model. The model developed is then applied in network of Pokhara City. A random set of destinations is selected and optimal route is found for that sample set of destinations. The model gives you the time required to travel between the destinations. Total time required to travel all destination is also calculated taking minimum time required to spend in a destination. Hence, the model can be used as a practical tool for optimizing tour time in a city network.

This model has a practical application in the field of tourism. Tourist agencies and tourist can use this model in trip planning. Apart from that, it can be used in the field of emergency supplies, telecommunication, tour planning for different purposes, vehicle routing and many more.

\section{REFERENCES}

A. Garcia, O. Arbelaitz, M. T. Linaza, P. Vansteenwegen, and W. Souriau (2010) Personalized Tourist Route Generation. ICWE'10 Proceedings of the 10th international conference on Current trends in web engineering, July 05 - 06, 2010, Pages 486-497

Dantzig, George B. (1963), Linear Programming and Extensions, Princeton, NJ: Princeton UP, pp. 545-7, ISBN 0-691-08000-3, sixth printing, 1974.

Golden, B., Levy, L., Vohra, R., 1987. The orienteering problem. Naval Research Logistics 34, 307-318.

I. M. Chao, B. L. Golden, E. A. Wasil (1996) Theory and Methodology-The team orienteering problem. European Journal of Operational Research 88 (1996) 464-474

Keller, P.C. (1989), "Algorithms to solve the orienteering problem: A comparison", European Journal of Operational Research 41, 224-231.

Laporte, G., and Martelio, S. (1990), "The selective traveling salesman problem", Discrete applied Mathematics 26, 193- 207.

M. Mataija, M. Rakamarić Šegić, F. Jozić: Solving the travelling salesman problem using the Branch and Bound method, Zbornik Veleučilišta u Rijeci, Vol. 4 (2016.), No. 1, pp. 259-270

P. Vansteenwegen and D. V. Oudheusden (2007). The Mobile Tourist Guide: An OR Opportunity. OR Insight Vol. 20 Issue 3 July - September 2007. 